\title{
EFEITO RESIDUAL DO LODO DE ESGOTO ALCALINIZADO EM ATRIBUTOS QUÍMICOS E GRANULOMÉTRICOS DE UM CAMBISSOLO HÚMICO ${ }^{1}$
}

\section{RESIDUAL EFFECT OF SEWAGE SLUDGE ON SOME CHEMICAL AND GRANULOMETRIC INCEPTISOL ATTRIBUTES}

\author{
Claudia de Azevedo BARCELAR ${ }^{2}$ \\ Aline Alinéia ROCHA ${ }^{3}$ \\ Marcelo Ricardo de LIMA ${ }^{4}$ \\ Marcos POHLMANN ${ }^{5}$
}

\begin{abstract}
RESUMO
O objetivo do presente trabalho foi avaliar o efeito residual do lodo de esgoto alcalinizado em atributos químicos e granulométricos do solo. Implantou-se um experimento no ano de 1995, em um CAMBISSOLO HÚMICO Distrófico gleico, localizado no Centro de Estações Experimentais do Canguiri - UFPR, em Pinhais, PR. Utilizaram-se os seguintes tratamentos: sem fertilização, fertilização mineral e fertilização orgânica com lodo de esgoto alcalinizado (32, 64 e $\left.96 \mathrm{~m}^{3} \cdot \mathrm{ha}^{-1}\right)$, com quatro repetições. As amostras utilizadas nesse estudo foram coletadas em 1998 nas profundidades de $0-5,5-10,10-20$ e $20-40 \mathrm{~cm}$, sendo realizadas as seguintes determinações: $\mathrm{Ca}, \mathrm{Mg}, \mathrm{K}$ e Al trocáveis, $\mathrm{H}+\mathrm{Al}, \mathrm{P}$ disponível, $\mathrm{C}$ orgânico, $\mathrm{pH}$ em $\mathrm{CaCl}_{2}, \mathrm{CTC}$ da matéria orgânica, da argila e total. Avaliaram-se, também, os conteúdos de areia, silte e argila. Não houve diferença significativa para o teor de $C$ orgânico nas profundidades estudadas e para o Ca nas profundidades de 0-5 e 10-20 cm. Não houve efeito das doses de lodo, na profundidade de $0-5 \mathrm{~cm}$, nos teores de Mg e K trocáveis, $\mathrm{P}$ disponível, $\mathrm{H}+\mathrm{Al}$, e para CTC total, da argila e orgânica. Os valores de pH aumentaram com a adição de lodo nas profundidades de 0-5 e 5-10 cm.
\end{abstract}

Palavras-chave: lodo de esgoto, capacidade de troca de cátions (CTC), fertilidade do solo.

\begin{abstract}
The purpose of this work was to evaluate the residual effect of sewage sludge on chemical and granulometric Inceptisol attributes. To achieve this purpose an experiment has been conducted since 1995 at the Canguiri Experimental Station Center, in Pinhais, PR. The selected treatments were no fertilization, mineral fertilization, and organic fertilization with sewage sludge (32, 64 and $\left.96 \mathrm{~m}^{3} . \mathrm{ha}^{-1}\right)$, with four replications. The soil samples were collect in 1998 at the following depths: $0-5,5-10,10-20$ and $20-40 \mathrm{~cm}$. The evaluations consisted of: exchangeable $\mathrm{Ca}, \mathrm{Mg}, \mathrm{K} \mathrm{Al}$, $\mathrm{H}+\mathrm{Al}$; available $\mathrm{P}$; organic $\mathrm{C}$; $\mathrm{pH}$ in $\mathrm{CaCl}_{2}$; organic matter, total and mineral $\mathrm{CEC}$; sand, loam and clay. No significant results were observed in organic $\mathrm{C}$ on the studied depth, or exchangeable $\mathrm{Ca}$ on the depth 0-5 and 10-20 cm. There was no significant effect of the sludge on exchangeable $\mathrm{Mg}$ and $K$, available $P, H+A l$, and total, clay and organic CEC. The $\mathrm{pH}$ value increased with addition of sewage sludge on the depth 0-5 and 5-10 $\mathrm{cm}$.
\end{abstract}

Key words: sewage sludge, cation exchange capacity (CEC), soil fertility.

1 Trabalho apresentado na $24^{a}$ Reunião Brasileira de Fertilidade do Solo e Nutrição de Plantas, Santa Maria, RS, OUT/2000.

2 Acadêmica do Curso de Engenharia Florestal da UFPR, Bolsista PIBIC UFPR/TN.

Acadêmica do Curso de Engenharia Florestal da UFPR, Bolsista PET/CAPES.

${ }^{4}$ Engenheiro Agrônomo, M. Sc., Universidade Federal do Paraná, Professor Assistente, Rua dos Funcionários, 1540, CEP

80035-050, Curitiba, PR. E-mail: mrlima@agrarias.ufpr.br @- Autor para correspondência.

${ }^{5}$ Engenheiro Agrônomo. 


\section{INTRODUÇÃO}

O lodo de esgoto é um resíduo que apresenta grande potencial de aproveitamento agrícola e florestal, quer como condicionante das propriedades físicas, químicas e biológicas do solo, quer como fonte de nutrientes para as plantas cultivadas, tendo em vista sua composição química [17]. Por essas e outras razões o aproveitamento agrícola do lodo de esgoto tem crescido muito nos países industrializados [15].

Segundo Outwater (1994) [19] a alternativa da reciclagem agrícola e florestal do lodo de esgoto transforma um resíduo em insumo, trazendo vantagens indiretas ao homem e ao ambiente, uma vez que pode: reduzir os efeitos adversos à saúde humana causados pela incineração, diminuir a dependência de fertilizantes químicos e melhorar o balanço de $\mathrm{CO}_{2}$ atmosférico. Além disso a utilização do lodo de esgoto pode melhorar a agregação das partículas, a infiltração e a retenção de água [3 e 13], e a aeração do solo [3, 6]. Observa-se, também, que a decomposição do lodo de esgoto gera agentes complexantes que facilitam a solubilização, no solo, de fosfatos combinados a ferro e alumínio, bem como libera, gradualmente, nutrientes essenciais às plantas, como $\mathrm{N}, \mathrm{P}, \mathrm{S}[6$, 10].

O lodo de esgoto tratado apresenta baixa contaminação, sendo um fertilizante de boa qualidade e de baixo custo. Pode ser usado na melhoria da produção florestal e agrícola e recuperação de áreas degradadas [1].

Silva et al. (1995) [21] verificaram que a aplicação do lodo de esgoto em solo sob soqueira de cana-de-açúcar proporcionou aumento do $\mathrm{pH}$, da soma de bases e da saturação por bases, da capacidade de troca de cátions (CTC) e da disponibilidade de $\mathrm{B}, \mathrm{Cd}, \mathrm{Cr}$, $\mathrm{Ni}$ e $\mathrm{Pb}$ e neutralização de parte da acidez potencial. Além disso, atuou como fonte de $\mathrm{Ca}, \mathrm{Mg}, \mathrm{P}$, Cu e $\mathrm{Zn}$.

A matéria orgânica, comum no lodo de esgoto, retém, em forma trocável, cátions essenciais e não essenciais às plantas. Isso evita ou reduz muito as perdas dos mesmos por percolação na água gravitacional [16].

O presente experimento teve como objetivo avaliar o efeito residual do lodo de esgoto alcalinizado em alguns atributos químicos e granulométricos do solo.

\section{METODOLOGIA}

A área experimental (CAMBISSOLO HÚMICO Distrófico gleico) pertence à Fazenda do Canguiri, da Universidade Federal do Paraná (UFPR) e localiza-se no município de Pinhais, PR $\left(25^{\circ} 25^{\prime}\right.$ de latitude Sul e $49^{\circ} 08^{\prime}$ de longitude Oeste e com altitude de $920 \mathrm{~m}$ ). A região possui um clima do tipo $\mathrm{Cfb}$ (subtropical úmido mesotérmico), segundo a classificação de Köppen [12]. O solo (0$20 \mathrm{~cm}$ ) antes da implantação do experimento apresentava os seguintes atributos:
$\mathrm{pH}\left(\mathrm{CaCl}_{2}\right)=5,3 ; \quad \mathrm{Al}^{+3}=0,0 \mathrm{mmol}_{\mathrm{c}} \cdot \mathrm{dm}^{-3} ; \quad \mathrm{H}+\mathrm{Al}=$ $54 \mathrm{mmol}_{\mathrm{c}} \cdot \mathrm{dm}^{-3} ; \quad \mathrm{Ca}+\mathrm{Mg}=117 \mathrm{mmol}_{\mathrm{c}} \cdot \mathrm{dm}^{-3} ; \quad \mathrm{K}=$ $1,5 \mathrm{mmol}_{\mathrm{c}} \cdot \mathrm{dm}^{-3}$ e C orgânico $=35 \mathrm{~g} \cdot \mathrm{dm}^{-3}$.

O lodo de esgoto foi aplicado no solo em 1995, sendo que não houve a aplicação do mesmo nos anos subseqüentes [9]. A área foi cultivada no sistema de plantio direto com as culturas de milho (safras de verão de 1995, 1996 e 1997) e aveia (inverno de 1997), ficando em pousio nos invernos de 1995 e 1996.

Os tratamentos utilizados foram: sem fertilização (testemunha), fertilização mineral $\left(60 \mathrm{~kg} \cdot \mathrm{ha}^{-1}\right.$ de $\mathrm{N}, 50 \mathrm{~kg} \cdot \mathrm{ha}^{-1}$ de $\mathrm{P}_{2} \mathrm{O}_{5}$ e $45 \mathrm{~kg} \cdot \mathrm{ha}^{-1}$ de $\left.\mathrm{K}_{2} \mathrm{O}\right)$ e adubação orgânica com lodo de esgoto alcalinizado ( $90 \%$ de umidade), proveniente da Estação Belém de Tratamento de Esgotos, da SANEPAR, nas doses de 32, 64 e $96 \mathrm{~m}^{3}$.ha-1. A dose de $32 \mathrm{~m}^{3}$.ha ${ }^{-1}$ corresponde aproximadamente à dosagem de $\mathrm{N}$ do tratamento com fertilização mineral. O lodo de esgoto foi distribuído a lanço sobre a superfície e incorporado ao solo, fazendo-se uma aração seguida de gradagem.

$O$ delineamento experimental utilizado foi o de blocos ao acaso com quatro repetições. As parcelas experimentais totais foram vinte, cada uma com $255 \mathrm{~m}^{2}$.

Para avaliar o efeito residual a amostragem foi feita três anos após a aplicação do lodo de esgoto. Coletaram-se amostras de solo nas profundidades $0-5,5-10,10-20$ e $20-40 \mathrm{~cm}$, sendo retiradas vinte sub-amostras por parcela.

As amostras de solo foram secas em estufa a $60^{\circ} \mathrm{C}$, destorroadas e passadas em peneira de 2,0 mm de diâmetro. Nessa fração fez-se a análise granulométrica [8]. Foram determinados: $\mathrm{C}$ orgânico por colorimetria [22], pH em $\mathrm{CaCl}_{2}$ 0,01 mol.dm ${ }^{-3}$, $\mathrm{Ca}^{+2}, \mathrm{Mg}^{+2}$ e Al$^{+3}$ trocáveis extraídos por solução de $\mathrm{KCl} 1 \mathrm{~mol} . \mathrm{dm}^{-3}, \mathrm{H}+\mathrm{Al}$ por meio da correlação com o índice SMP, $\mathrm{K}^{+}$trocável e $\mathrm{P}$ disponível extraídos com

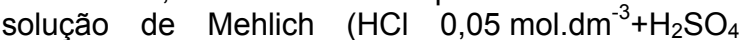
$0,0125 \mathrm{~mol}^{-\mathrm{dm}^{-3}}$ ) [20]. Na profundidade de $0-5 \mathrm{~cm}$, a CTC total e das argilas foram determinadas a pH 7,0 [5], sendo que, para a determinação da CTC das argilas, houve a eliminação da matéria orgânica pela da incineração das amostras a $350^{\circ} \mathrm{C}$ em mufla. Obteve-se a CTC da matéria orgânica pela diferença entre a CTC total e a CTC das argilas.

Os dados obtidos foram analisados estatisticamente e, quando necessário, as médias foram comparadas pelo teste de Tukey a $5 \%$.

\section{RESULTADOS E DISCUSSÃO}

$\mathrm{O} \mathrm{pH}$ em $\mathrm{CaCl}_{2}$ aumentou com a adição de lodo nas profundidades de $0-5$ e $5-10 \mathrm{~cm}$ (Tabela 1 ), enquanto a fertilização mineral resultou em valores significativamente menores. $\mathrm{O}$ aumento do $\mathrm{pH}$ do solo em $\mathrm{CaCl}_{2}$, determinado pela aplicação do lodo de esgoto alcalinizado, está associado a sua composição química, uma vez que o mesmo resulta da mistura com cal (CaO). Esses resultados estão de acordo com aqueles obtidos por Silva et al. (1995) [21], que verificaram que a aplicação do 
lodo de esgoto em soqueira de cana-de-açúcar proporcionou aumentos no $\mathrm{pH}$. Resultados semelhantes foram obtidos por Berton et al. (1989) [2], na cultura do milho. A inexistência de
$\mathrm{Al}^{+3}$ trocável nos diferentes tratamentos está associada aos altos valores de $\mathrm{pH}$ (Tabela 1), concordando com os resultados obtidos por Morais et al. (1996) [18].

TABELA 1 -Efeito residual de diferentes fertilizações nos valores médios de pH de um CAMBISSOLO HÚMICO, em Pinhais, PR, $1998^{1}$

\begin{tabular}{|c|c|c|c|c|c|c|c|}
\hline \multirow{3}{*}{$\begin{array}{l}\text { Profundidade } \\
\qquad(\mathrm{cm})\end{array}$} & \multicolumn{5}{|c|}{ Tratamentos } & \multirow{3}{*}{$\begin{array}{l}\mathrm{CV}^{2} \\
(\%)\end{array}$} & \multirow{3}{*}{$\begin{array}{c}\text { DMS }^{3} \\
(5 \%)\end{array}$} \\
\hline & \multirow{2}{*}{ Testemunha } & \multirow{2}{*}{$\begin{array}{c}\text { Fertilização } \\
\text { Mineral }\end{array}$} & \multicolumn{3}{|c|}{ Lodo de Esgoto } & & \\
\hline & & & $32 \mathrm{~m}^{3} \cdot \mathrm{ha}^{-1}$ & $64 \mathrm{~m}^{3} \cdot \mathrm{ha}^{-1}$ & $96 \mathrm{~m}^{3} \cdot \mathrm{ha}^{-1}$ & & \\
\hline $0-5$ & $5,6 \mathrm{bc}$ & $5,3 \mathrm{c}$ & $5,8 \mathrm{ab}$ & $5,8 \mathrm{ab}$ & $6,0 a$ & 2,9 & 0,36 \\
\hline $5-10$ & 5,6 bc & $5,4 \mathrm{c}$ & $5,9 a b$ & 6,0 a & $6,1 \mathrm{a}$ & 3,0 & 0,39 \\
\hline $10-20$ & $5,7 a b$ & $5,5 \mathrm{~b}$ & $5,9 a b$ & $5,9 a b$ & $6,1 \mathrm{a}$ & 3,6 & 0,46 \\
\hline $20-40$ & $5,3 a b$ & $5,1 \mathrm{~b}$ & $5,6 a b$ & 5,7 a & $5,8 \mathrm{a}$ & 4,3 & 0,52 \\
\hline
\end{tabular}

Os teores de $\mathrm{Ca}^{+2}$ trocável nas profundidades de $0-5$ e $10-20 \mathrm{~cm}$ (Tabela 2) não foram influenciados significativamente pelos tratamentos testados. Entretanto, as doses de 64 e $96 \mathrm{~m}^{3}$.ha-1, promoveram aumento significativo no teor de $\mathrm{Ca}^{+2}$ trocável no solo em relação à testemunha e à fertilização mineral, na profundidade $5-10 \mathrm{~cm}$. A dose de $32 \mathrm{~m}^{3} \cdot$ ha $^{-1}$ também promoveu aumento significativo no teor de $\mathrm{Ca}^{+2}$, em relação à fertilização mineral, na profundidade de $20-40 \mathrm{~cm}$ (Tabela 2). Esses resultados são concordantes com os de Silva et al. (1995) [21], Berton et al. (1989) [2], e Lourenço et al. (1996) [14], que verificaram que o lodo de esgoto atua como fonte de Ca.

TABELA 2 -Efeito residual de diferentes fertilizações nas médias de $\mathrm{Ca}$, $\mathrm{Mg}$ e $\mathrm{K}$ trocáveis, $\mathrm{P}$ disponível, $\mathrm{H}+\mathrm{Al}$, e C orgânico em um CAMBISSOLO HÚMICO, em Pinhais, PR, $1998^{1}$

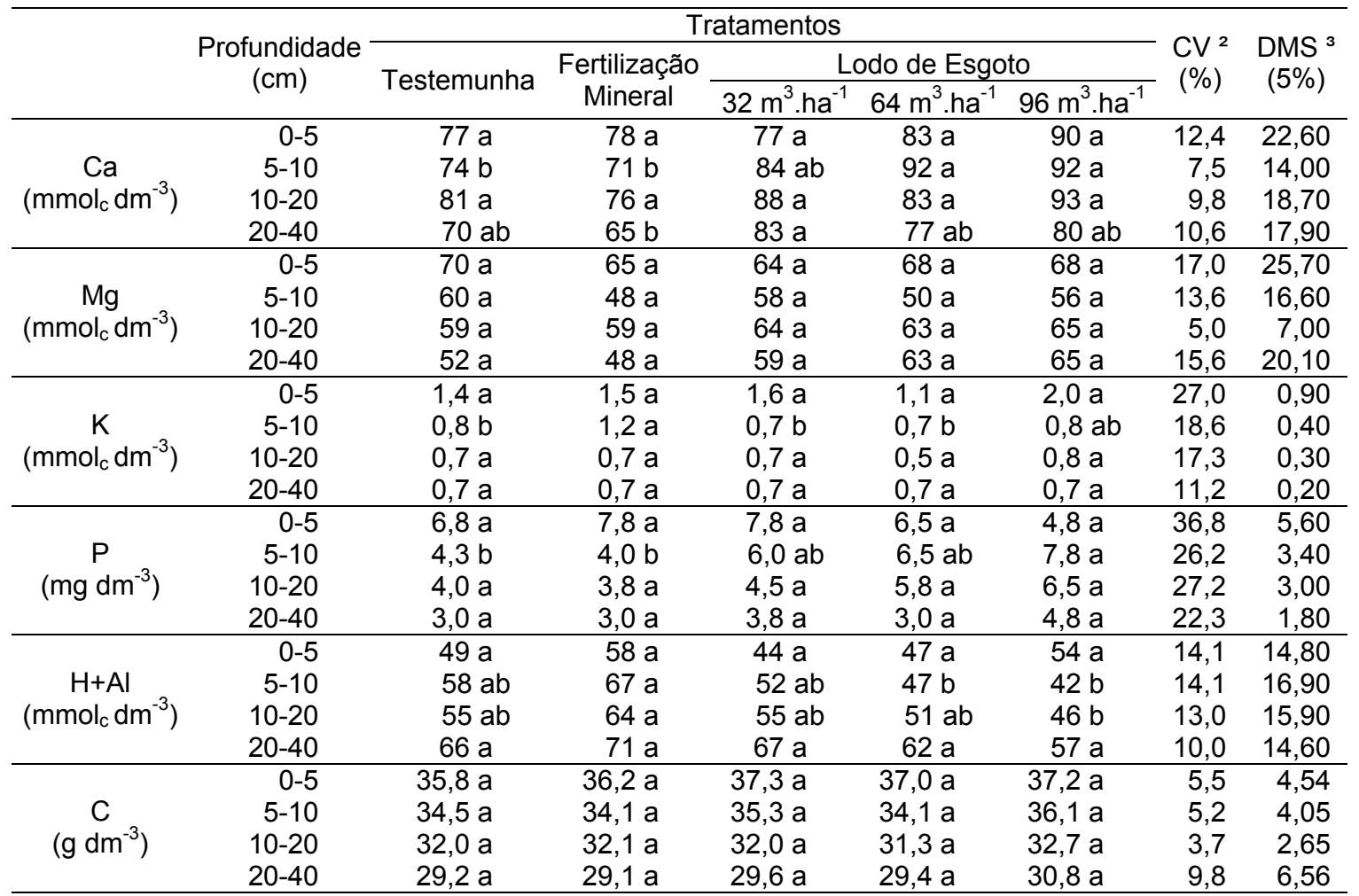

1 Médias seguidas por letras distintas na linha diferem entre si ao nível de significância de $5 \%$ pelo teste de Tukey

2 Coeficiente de variação

3 Diferença média significativa 
O teor de C orgânico não aumentou significativamente com o aumento das doses de lodo nas profundidades estudadas (Tabela 2). Esses resultados concordam com os obtidos por Da Ros et al. (1993) [7], que afirmaram que o efeito de doses de lodo sobre o teor de carbono do solo desaparece com o decorrer do tempo. Para esses autores, na dose de $32 \mathrm{~m}^{3}$.ha ${ }^{-1}$, o efeito sobre o $\mathrm{C}$ orgânico desaparece após 230 dias. Segundo eles a maior parte do C orgânico adicionado foi rapidamente decomposta pela ação dos microorganismos do solo. Os resultados discordam dos obtidos por Fiest et al. (1998)[11], que verificaram tendência na redução do teor de $\mathrm{C}$ orgânico nos tratamentos com lodo, porém, sem haver diferença significativa.

Os tratamentos não influenciaram significativamente $\mathrm{o}$ teor de $\mathrm{Mg}^{+2}$ trocável, independetemente da profundidade considerada
(Tabela 2). Segundo Silva et al. (1995) [21], o lodo de esgoto aumenta o de $\mathrm{Mg}^{+2}$ trocável do solo, porém esta situação não foi observada neste experimento.

Para os teores de $\mathrm{K}^{+}$trocável, $\mathrm{P}$ disponível e $\mathrm{H}+\mathrm{Al}$, na profundidade $0-5 \mathrm{~cm}$, não ocorreram variações significativas (Tabela 2 ). Esses resultados diferem daqueles obtidos por Silva et al. (1995) [21], que verificaram que a aplicação do lodo de esgoto atua como fonte de $\mathrm{P}$ em soqueira de cana-deaçúcar. Entretanto, na profundidade de $5-10 \mathrm{~cm}$, para a maior dose de lodo, o teor de $\mathrm{P}$ disponível foi significativamente maior que na testemunha. $\mathrm{Na}$ profundidade de $5-10$ e $10-20 \mathrm{~cm}, 0 \mathrm{H}+\mathrm{AL}$ foi significativamente inferior no tratamento com fertilização mineral (Tabela 2), o que está de acordo com os valores de $\mathrm{pH}\left(\mathrm{CaCl}_{2}\right)$ encontrados nestas profundidades (Tabela 1 ).

TABELA 3 -Efeito residual de diferentes fertilizações nas médias dos teores de areia, silte e argila em um CAMBISSOLO HÚMICO, em Pinhais, PR, $1998^{1}$

\begin{tabular}{|c|c|c|c|c|c|c|c|c|}
\hline & \multirow{3}{*}{$\begin{array}{l}\text { Profundidade } \\
\qquad(\mathrm{cm})\end{array}$} & \multicolumn{5}{|c|}{ Tratamentos } & \multirow{3}{*}{$\begin{array}{l}\mathrm{CV}^{2} \\
(\%)\end{array}$} & \multirow{3}{*}{$\begin{array}{l}\text { DMS }^{3} \\
(5 \%)\end{array}$} \\
\hline & & \multirow{2}{*}{ Testemunha } & \multirow{2}{*}{$\begin{array}{c}\text { Fertilização } \\
\text { Mineral }\end{array}$} & \multicolumn{3}{|c|}{ Lodo de Esgoto } & & \\
\hline & & & & $32 \mathrm{~m}^{3} \cdot \mathrm{ha}^{-1}$ & $64 \mathrm{~m}^{3} \cdot \mathrm{ha}^{-1}$ & $96 \mathrm{~m}^{3} \cdot \mathrm{ha}^{-1}$ & & \\
\hline \multirow{4}{*}{$\begin{array}{c}\text { Areia } \\
\left(\mathrm{g} \mathrm{kg}^{-1}\right)\end{array}$} & $0-5$ & 220 bc & $210 \mathrm{c}$ & $265 a$ & $255 a b$ & $280 \mathrm{a}$ & 17,0 & 35,6 \\
\hline & $5-10$ & $262 a$ & $249 a$ & $255 a$ & $271 \mathrm{a}$ & $246 a$ & 6,0 & 34,6 \\
\hline & $10-20$ & $241 \mathrm{a}$ & $238 a$ & $238 a$ & $239 a$ & 229 a & 5,9 & 31,4 \\
\hline & $20-40$ & 248 a & $242 \mathrm{a}$ & $243 a$ & 259 a & $242 a$ & 7,8 & 43,3 \\
\hline \multirow{4}{*}{$\begin{array}{c}\text { Silte } \\
\left(\mathrm{g} \mathrm{kg}^{-1}\right)\end{array}$} & $0-5$ & $355 a$ & $345 a$ & $280 \mathrm{bc}$ & $325 a b$ & $245 \mathrm{c}$ & 7,9 & 52,5 \\
\hline & $5-10$ & $333 a$ & $327 a$ & $325 a$ & $314 a$ & $309 a$ & 6,4 & 46,1 \\
\hline & $10-20$ & $274 b$ & $308 a$ & $307 a$ & $316 a$ & $301 a$ & 4,2 & 28,9 \\
\hline & $20-40$ & $297 a$ & $253 a$ & $302 a$ & $293 a$ & $303 a$ & 8,6 & 56,4 \\
\hline \multirow{4}{*}{$\begin{array}{c}\text { Argila } \\
\left(\mathrm{g} \mathrm{kg}^{-1}\right)\end{array}$} & $0-5$ & $425 a b$ & $445 a b$ & $455 a b$ & $420 \mathrm{~b}$ & $475 a$ & 5,4 & 54,3 \\
\hline & $5-10$ & $405 a$ & $425 a$ & $420 a$ & $415 a$ & $445 a$ & 5,9 & 56,4 \\
\hline & $10-20$ & $460 \mathrm{a}$ & $455 a$ & $455 a$ & $445 a$ & $450 a$ & 5,7 & 58,1 \\
\hline & $20-40$ & $455 b$ & $505 a$ & $455 b$ & $450 \mathrm{~b}$ & $455 b$ & 3.9 & 41.2 \\
\hline
\end{tabular}

1 Médias seguidas por letras distintas na linha diferem entre si ao nível de significância de $5 \%$ pelo teste de Tukey

2 Coeficiente de variação

3 Diferença média significativa

$\mathrm{Na}$ profundidade de $0-5 \mathrm{~cm}$, os valores de areia nos tratamentos que receberam lodo foram significativamente maiores que no tratamento com fertilização mineral, bem como os valores de silte foram maiores nos tratamentos testemunha e fertilização mineral em relação às doses de 32 e $96 \mathrm{~m}^{3}$.ha ${ }^{-1}$ de lodo (Tabela 3).

Não houve diferença estatística nos valores da CTC da matéria orgânica, de argilas e total, nos diferentes tratamentos, na profundidade de $0-5 \mathrm{~cm}$ (Tabela 4). Silva et al. (1995) [21] e Braccini et al. (1995) [4] verificaram que com a aplicação de lodo de esgoto e resíduos orgânicos aumentou significativamente a CTC total do solo. Os resultados obtidos nesse trabalho (Tabela 4) contradizem aqueles apresentados pelos autores, porém deve-se considerar que são estudos de curta duração.

TABELA 4 - Efeito residual de diferentes fertilizações nas médias da CTC orgânica, de argila e total em um CAMBISSOLO HÚMICO na profundidade de 0-5 cm, em Pinhais, PR. $1998^{1}$

\begin{tabular}{|c|c|c|c|c|c|c|c|}
\hline \multirow{3}{*}{$\begin{array}{c}\text { CTC } \\
\left(\mathrm{mmol}_{\mathrm{c}} \mathrm{kg}^{-1}\right)\end{array}$} & \multicolumn{5}{|c|}{ Tratamentos } & \multirow{3}{*}{$\begin{array}{l}\mathrm{CV}^{2} \\
(\%)\end{array}$} & \multirow{3}{*}{$\begin{array}{c}\text { DMS }^{3} \\
(5 \%)\end{array}$} \\
\hline & \multirow{2}{*}{ Testemunha } & \multirow{2}{*}{$\begin{array}{c}\text { Fertilização } \\
\text { Mineral }\end{array}$} & \multicolumn{3}{|c|}{ Lodo de Esgoto } & & \\
\hline & & & $32 \mathrm{~m}^{3} \cdot \mathrm{ha}^{-1}$ & $64 \mathrm{~m}^{3} \cdot \mathrm{ha}^{-1}$ & $96 \mathrm{~m}^{3} \cdot \mathrm{ha}^{-1}$ & & \\
\hline Orgânica & $180 \mathrm{a}$ & $187 \mathrm{a}$ & $172 \mathrm{a}$ & $173 \mathrm{a}$ & $181 \mathrm{a}$ & 10,7 & 42,9 \\
\hline Argilas & $83 a$ & $74 \mathrm{a}$ & $81 \mathrm{a}$ & 85 a & 82 a & 10,7 & 19,5 \\
\hline Total & 263 a & $260 \mathrm{a}$ & $253 a$ & 258 a & 263 a & 8,3 & 48,3 \\
\hline
\end{tabular}




\section{CONCLUSÕES}

Com a adição de lodo de esgoto no solo aumentou-se $\mathrm{o} \mathrm{pH}$, nas profundidades de 0-5 e 5$10 \mathrm{~cm}$. Não houve efeito residual significativo para os teores de $\mathrm{C}$ orgânico nas profundidades estudadas e para $\circ \mathrm{Ca}^{+2}$ trocável nas profundidades $0-5$ e $10-20 \mathrm{~cm}$. Independente da profundidade considerada não foram observadas variações significativas para os valores de CTC da fração orgânica e total em função dos tratamentos estudados.

\section{AGRADECIMENTOS}

Agradecemos aos professores Nerilde Favaretto e Cícero Deschamps pela cessão da área para estudo e à Maria Aparecida Carvalho dos Santos pelo auxílio nas atividades de laboratório.

\section{REFERÊNCIAS}

[1] ANDREOLI, C. V.; BARRETO, C. L. G.; BONNET, B. R. P.; FERNANDES, F.; NERY, A. C. Tratamento e disposição final de lodo de esgoto no Paraná. Sanare, Curitiba, v.1, n.1, p. 10-15, 1994.

[2] BERTON, R. S.; CAMARGO, O. A.; VALADARES, J. M A. S. Absorção de nutrientes pelo milho em resposta à adição de lodo de esgoto a cinco solos paulistas. Revista Brasileira de Ciência do Solo, Campinas v.13, n.2, p. 187-192, 1989

[3] BONNET, B. R. P. Diagnóstico de situação e proposição de sistema de monitoramento dos impactos ambientais causados pela reciclagem agrícola do lodo de esgotos. Curitiba, 1995. $73 \mathrm{f}$ Monografia (Especialização no Curso de PósGraduação Lato Sensu em Análise Ambiental) - Setor de Tecnologia, Universidade Federal do Paraná.

[4] BRACCINI, A. L.; BRITO, C. M.; PÔNZIO, J. B.; MORETTI, C. L.; LOURES, E. G. Efeito da aplicação de resíduos orgânicos com diferentes relações $\mathrm{C} / \mathrm{N}$ sobre algumas características químicas de um Latossolo Vermelho Escuro. Revista Ceres, Viçosa, v.42, n.244, p. 671-684, 1995

[5] CAMARGO, O. A.; MONIZ, A. C.; JORGE, J. A.; VALADARES, J. M. S. L. Métodos de análise química, mineralógica e física de solos do Instituto Agronômico de Campinas. Campinas: Instituto Agronômico, 1986. 94 p. (IAC. Boletim Técnico 106).

[6] CARVAlHO, P. C. T.; BARRAL, M. F. Aplicação de lodo de esgoto como fertilizante. Fertilizantes, São Paulo, v.3, n.3, p. 3-5, 1981

[7] DA ROS, C. O.; AITA, C.; CERETTA, C. A.; FRIES, M R. Lodo de esgoto: efeito imediato no milheto e residual na associação aveia-ervilhaca. Revista Brasileira de Ciência do Solo, Campinas, v.17, n.2, p. 257-261, 1993.

[8] EMBRAPA - Empresa Brasileira de Pesquisa Agropecuária / Centro Nacional de Pesquisa de Solos. Manual de métodos de análise de solos. 2.ed. Rio de Janeiro: EMBRAPA, 1997. 212 p. (Documentos 01).
[9] FAVARETTO, N: DECHAMPS, C.: MOTTA, A C. V. Efeito do lodo de esgoto na fertilidade do solo e no crescimento e produtividade do milho. In: CONGRESSO BRASILEIRO DE CIÊNCIA DO SOLO 23., Rio de Janeiro,1997. Resumos. Rio de Janeiro: SBCS, 1997. p. 143.

[10]FERNANDES, F.; ANDRAUS, S.; ANDREOLI, C. V.; BONNET, B. R. P.; BORGES, J. C.; CANTO, L. A.; MEDEIROS, M. L. B. Eficiência dos processos de desinfecção do lodo da ETE-Belém com vista a seu uso agrícola. Sanare, Curitiba, v.5, n.5, p. 46-58, 1996.

[11]FIEST, L. C.; ANDREOLI, C. V.; MACHADO, M. A. M. Efeitos da aplicação do lodo de esgoto nas propriedades físicas do solo. Sanare, Curitiba, v.9, n.9, p. 48-57, 1998.

[12] IAPAR - Instituto Agronômico do Paraná. A cultura do milho no Paraná. Londrina: IAPAR, 1994. 217 p. (Circular, 68).

[13]JORGE, J. A.; CAMARGO, O. A.; VALADARES, J. M. A. S. Condições físicas de um Latossolo Vermelho Escuro quatro anos após aplicação de lodo de esgoto e calcário. Revista Brasileira de Ciência do Solo, Campinas, v.15, n.3, p. 237-240, 1991.

[14]LOURENÇO, R. S.; ANJOS, A. R. M.; LIBARDI, P. L.; MEDRADO, M. J. S. Efeito do lodo de esgoto na produtividade de milho e feijão, no sistema de produção da bracatinga. Sanare, Curitiba, v.5, n.5, p. 90-92, 1996.

[15]LUE-HING, C.; PIETZ, R. I.; GRANATO, T. C.; GSCHWIND, J.; ZENZ, D. R. Overview of the past 25 years: operator's perspective. In: Sewage sludge: land utilization and environmental. Madison: SSSA, 1994. p. 7-16.

[16]MELLO, F. A. F., BRASIL SOBRINHO, M. O. C.; ARZOLLA, S.; SILVEIRA, R. I.; COBRA NETTO, A.; KIEHL, J. C. Fertilidade do solo. 3.ed. São Paulo: Nobel, 1989. $400 \mathrm{p}$.

[17]MELLO, W. J.; MARQUES, M. O.; SANTIAGO, G.; CHELLI, R. A.; LEITE, S. A. S. Efeito de doses crescentes de lodo de esgoto sobre frações da matéria orgânica e CTC de um Latossolo cultivado com canade-açúcar. Revista Brasileira de Ciência do Solo, Campinas. v.18, n.3, p. 449-455, 1994.

[18]MORAIS, S. M. de J.; ATAIDES, P. R. V.; GARCIA, D. C.; KURTZ, F. C.; OLIVEIRA, O. S.; WATZLAWICK, L. F. Uso do lodo de esgoto da Corsan - Santa Maria (RS), comparada com outros substratos orgânicos. Sanare, Curitiba, v.6, n.6, p. 44-49, 1996.

[19] OUTWATER, A. B. Reuse sewage sludge and minor wastewater residuals. Saint Louis: Lewis Publishers, 1994. 179 P.

[20]PAVAN, M. A.; BLOCH, M. F.; ZEMPULSKI, H. C.; MIYAZAWA, M.; ZOCOLER, D. C. Manual de análise química de solo e controle de qualidade. Londrina: IAPAR, 1992. 40 p. (Circular, 76).

[21]SILVA, F. C.; BOARETTO, A. E.; BERTON, R. S.; ZOTELLI, H. B.; PEXE, CA. Características agrotecnológicas, teores de nutrientes e de metais pesados em cana-de-açúcar (soqueira), cultivada em solo adubado com lodo de esgoto. In: CONGRESSO BRASILEIRO DE CIÊNCIA DO SOLO 25., 1995, Viçosa. Anais. Viçosa: SBSC/UFV, 1995. p. 22792281.

[22]TEDESCO, M. J.; VOLKWEISS, S. J.; BOHNEN, H. Análises de solo, plantas e outros materiais. Porto Alegre: Universidade Federal do Rio Grande do Sul, Departamento de Solos, 1985. $174 \mathrm{p}$. 